\title{
Wearable Experience Capture for Episodic Memory Support
}

\author{
Matthew L. Lee \& Anind K. Dey \\ Human-Computer Interaction Institute, Carnegie Mellon University \\ [mllee, anind] @cs.cmu.edu
}

\begin{abstract}
People with recent episodic memory impairment (EMI) such as individuals with Alzheimer's disease suffer from the lack of awareness of their recent experiences. We designed a wearable lifelogging system that allows these individuals to automatically and passively capture their experiences, select and annotate the most salient memory cues from the lifelog, and present these cues to support reminiscence of the experience with a self-guided, memory-exercising interaction.
\end{abstract}

\section{Introduction}

Episodic memory impairment (EMI) is the main symptom associated with Alzheimer's disease, a common neurodegenerative disease that affects over 26 million people worldwide [1]. Recent episodic memory supports our sense of self by enabling us to mentally travel back in time and relive our pleasant experiences and to be socially and physically engaged in our lives. EMI can lead to a loss in autonomy and control in individuals' lives, resulting in feelings of uncertainty, irritation, and frustration as they attempt to compensate for their memory loss but repeatedly fail.

\section{Lifelogging Systems as Memory Support}

Ubiquitous lifelogging systems use wearable or embedded sensor technologies such as cameras, audio recorders, location trackers, and physiological sensors to passively and automatically record a user's personal experiences. Lifelogging technologies allow people with EMI to automatically record, review, and thus regain an awareness of meaningful personal experiences in their lives to maintain their sense of self. Sellen et al. [2] showed that episodic details from a visual "lifelog" can be presented to users as memory cues to assist them in remembering the details of the original experience. However, lifelogging technologies often automatically capture an overwhelmingly large amount of data that can be both tedious and difficult to review, especially for people with cognitive impairment. To be most effective in supporting memory, lifelogging systems need to select the most important information to present to the user in an engaging way.

\section{System Design}

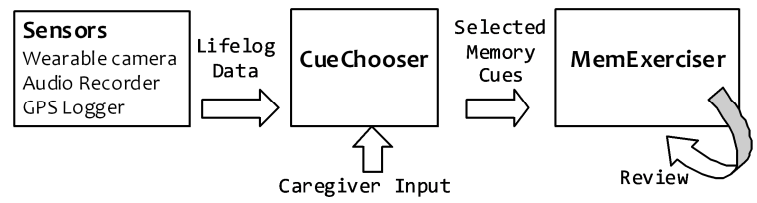

Figure 1. Our system consists of three steps: Capture, Selection, and Review.

We designed a lifelogging system that follows a three-step process of capture, cue selection, and review to support recollection of episodic memories (Figure 1). Using a combination of wearable sensors, the system passively records experiences of the user's choosing. Then, the caregiver uses the CueChooser application to construct a narrative of the experience by selecting and annotating the best memory cues from the recorded lifelog data, with the help from content and context analysis. Finally, with the MemExerciser application, the caregiver-chosen cues are presented to the person with EMI in a way that maximizes the opportunities for them to think deeply about the cues to remember associated details on their own without requiring repeated live help from their caregiver.

Our proof-of-concept capture system records photographs, ambient voices and sounds, and location information from the user's experiences using three devices (Figure 3): the Microsoft SenseCam, an offthe-shelf voice recorder, and a GPS logger. The SenseCam [3] is a wearable digital camera worn at chest level that automatically takes photos. The SenseCam can use various sensors such as a three-axis accelerometer, temperature, light, and infrared sensors to determine "good" opportunities to take a photo. In addition to photos, other cues for memory include 
ambient voices and sounds [4] and location information [5]. Ambient voices and sounds from the user's experience were recorded using an Olympus WS-310M voice recorder, placed in the user's shirt top pocket. The recorder was able to record the voices of the user and nearby people. GPS location information was also recorded every 1 to 5 seconds using a Wintec WBT201 GPS logger.

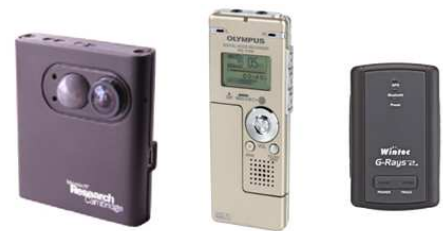

Figure 3. A wearable digital camera, audio recorder, and GPS logger capture the user's experience.

Our lifelogging system, despite only spanning three primary modalities (visual, audio, and location), can still capture an overwhelming amount of data from an experience. The types of cues that need to be extracted are based on the type of experience [5]. Our approach leverages automated content and context analysis (e.g., face detection with photos for peoplebased experiences and localization using GPS for location-based experiences) to extract potentially helpful memory cues from the lifelog and filter it down to a more manageable size. We also leverage the expertise of the caregiver of the person with EMI to hand select meaningful memory cues from the filtered content to present to the person with EMI. The caregiver constructs a slideshow narrative of photos, sounds, and annotations.

MemExerciser consists of an application (Figure 2) installed on a Toshiba M200 tablet PC so that it mimics a picture frame that the person with memory impairment can pick up and use without having to worry about operating a computer or bothering their caregiver. MemExerciser reveals each photo, the associated recorded sounds, and the caregiver's annotations one at a time so that it follows a similar cueing process [5] used by the caregiver in the absence of any technological support.

\section{Evaluation and Summary}

We conducted a preliminary evaluation with three older adults with EMI and their spouses (their primary caregiver). Each participant used the system to record an interesting outing and reviewed the caregiverauthored slideshow roughly every other day for the following two weeks. Participants felt the capture devices were unobtrusive and were mostly unnoticed

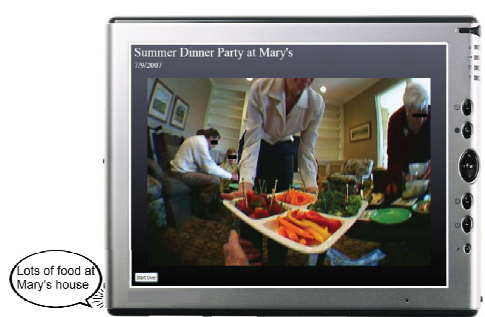

Figure 2. MemExerciser progressively reveals cues to support memory reminiscence.

by others. However, caregivers remarked that they were worried that they did not know whether they switched on the devices properly. Each experience lasted about 2-3 hours with an average of 176 photos taken. After the experience, caregivers enjoyed looking through the photos when constructing the slideshow, using the selected photos to browse content from the rest of the lifelog. Caregivers usually selected photos that they thought their spouse would recognize as a way to trigger their memory. Caregivers spent about an hour to construct the slideshow that averaged around 27 photos with approximately $40 \%$ of them suggested by the system. Participants enjoyed having the ability to refresh their memory of the experience on their own without bothering the caregiver. The combination of automatically captured photos, ambient audio, and caregiver's annotations provided sufficient cognitive scaffolding to support the participants' ability to remember details that they did not initially recall.

Our system not only was successful at capturing a personal experience, but it also provided a way to structure the information in such a way that the person with EMI could best utilize it to reminisce about recent events and reduce the burden on their caregiver.

\section{References}

[1] American_Health_Assistance_Foundation, "http://www.ahaf.org/alzdis/about/adabout.htm."

[2] A. J. Sellen, A. Fogg, M. Aitken, S. Hodges, C. Rother, and K. Wood, "Do life-logging technologies support memory for the past?: an experimental study using sensecam," Proc. CHI 2007, pp. 81-90, 2007.

[3] S. Hodges, L. Williams, E. Berry, S. Izadi, J. Srinivasan, A. Butler, G. Smyth, N. Kapur, and K. Wood, "SenseCam: a Retrospective Memory Aid," Proc. UBICOMP, pp. 81 - 90, 2006.

[4] S. Vemuri, C. Schmandt, and W. Bender, "iRemember: a personal, long-term memory prosthesis," Proc. CARPE 2006, pp. 65-74, 2006.

[5] M. L. Lee and A. K. Dey, "Providing Good Memory Cues for People with Episodic Memory Impairment," Proc. ASSETS 2007, pp. 131 - 138 2007. 